\title{
Diagnostic accuracy of 64-slice computed tomography coronary angiography for the detection of in-stent restenosis: A meta-analysis
}

\author{
Nazario Carrabba, MD, , Joanne D. Schuijf, PhD, ${ }^{b}$ Fleur R. de Graaf, MD, \\ Guido Parodi, $M D,{ }^{\text {a }}$ Erica Maffei, $M D,{ }^{c}$ Renato Valenti, $M D,{ }^{a}$ \\ Alessandro Palumbo, MD, ${ }^{\mathrm{c}}$ Annick C. Weustink, MD, ${ }^{\mathrm{d}}$ Nico R. Mollet, MD, PhD, ${ }^{\mathrm{d}}$ \\ Gabriele Accetta, PhD, ${ }^{\mathrm{e}}$ Filippo Cademartiri, MD, PhD, ${ }^{\mathrm{c}, \mathrm{d}}$ David Antoniucci, MD, ${ }^{\mathrm{a}}$ \\ and Jeroen J. Bax, MD, PhD ${ }^{\mathrm{b}}$
}

Background. We sought to evaluate the diagnostic accuracy of 64-slice multi-detector row computed tomography (MDCT) compared with invasive coronary angiography for in-stent restenosis (ISR) detection.

Methods. MEDLINE, Cochrane library, and BioMed Central database searches were performed until April 2009 for original articles. Inclusion criteria were (1) 64-MDCT was used as a diagnostic test for ISR, with $>\mathbf{5 0 \%}$ diameter stenosis selected as the cut-off criterion for significant ISR, using invasive coronary angiography and quantitative coronary angiography as the standard of reference; (2) absolute numbers of true positive, false positive, true negative, and false negative results could be derived. Standard meta-analytic methods were applied.

Results. Nine studies with a total of 598 patients with 978 stents included were considered eligible. On average, $9 \%$ of stents were unassessable (range 0-42\%). Accuracy tests with $95 \%$ confidence intervals (CIs) comparing 64-MDCT vs invasive coronary angiography showed that pooled sensitivity, specificity, positive and negative likelihood ratio (random effect model) values were: 86\% (95\% CI 80-91\%), 93\% (95\% CI 91-95\%), 12.32 (95\% CI 7.26-20.92), 0.18 $(95 \%$ CI 0.12-0.28) for binary ISR detection. The symmetric area under the curve value was 0.94, indicating good agreement between 64-MDCT and invasive coronary angiography.

Conclusions. 64-MDCT has a good diagnostic accuracy for ISR detection with a particularly high negative predictive value. However, still a relatively large proportion of stents remains uninterpretable. Accordingly, only in selected patients, 64-MDCT may serve as a potential alternative noninvasive method to rule out ISR. (J Nucl Cardiol 2010;17:470-8.)

Key Words: Meta-analysis $\bullet$ in-stent restenosis $\cdot$ computed tomography coronary angiography

\section{INTRODUCTION}

Coronary stent implantation is increasingly performed in the treatment of significant coronary artery disease and has significantly reduced the occurrence of

From the Division of Cardiology, ${ }^{\mathrm{a}}$ Careggi Hospital, Florence, Italy; Department of Cardiology, ${ }^{\mathrm{b}}$ Leiden University Medical Center, Leiden, The Netherlands; Department of Radiology, ${ }^{\mathrm{c}}$ Academic Hospital, Parma, Italy; Department of Cardiology and Radiology, ${ }^{\mathrm{d}}$ Erasmus Medical Centre, Rotterdam, The Netherlands; and Biostatistics Unit, ${ }^{\mathrm{e}}$ ISPO, Cancer Prevention and Research Institute, Florence, Italy.

Disclosures: Dr de Graaf is co-supported by the Dutch Technology Foundation STW (Utrecht, the Netherlands), Applied Science Division of NWO and the Technology Program of the Ministry of Economic Affairs, Grant No. 10084. Dr Bax has research grants restenosis as compared with balloon angioplasty. ${ }^{1,2}$ Moreover, with the recent introduction of drug-elutingstents (DES), the occurrence of in-stent restenosis (ISR) has further decreased. ${ }^{3,4}$ Nonetheless, even after DES

from Medtronic, Boston Scientific, BMS Medical Imaging, St. Jude Medical, GE Healthcare, Biotronik, and Edwards Lifesciences.

Received for publication Dec 23, 2009; final revision accepted Feb 28, 2010.

Reprint requests: Nazario Carrabba, MD, Division of Cardiology, Careggi Hospital, V.le Morgagni, 85, Florence, Italy; carddept@ tin.it. $1071-3581 / \$ 34.00$

Copyright $\odot 2010$ by the The Author(s). This article is published with open access at Springerlink.com doi:10.1007/s12350-010-9218-2 
implantation, excessive neo-intimal hyperplasia resulting in partial or complete ISR may still occur. Accordingly, in patients presenting with recurrent chest pain following DES implantation, invasive coronary angiography remains frequently indicated to evaluate the presence of ISR. However, considering the fact that a substantial number of these invasive coronary angiograms are not followed by intervention, the need for a noninvasive alternative approach for ISR detection is evident. To this end, stress tests may be used to assess patients with suspicion of ISR. However, the diagnostic accuracy of stress tests for ISR detection is moderate, ${ }^{5}$ and direct stent visualization would be preferred. With previous 4- and 16-slice multi-detector row computed tomography (MDCT) systems, MDCT was of limited value in the assessment and follow-up of patients with coronary stents, due to the frequent occurrence of motion and blooming artefacts. ${ }^{6,7}$ With the introduction of 64-slice systems, some of these limitations have been partially overcome due to increased temporal resolution (due to increased gantry rotation speed), increased spatial resolution and enhanced craniocaudal coverage. Although initial data obtained with 64-MDCT appear promising, more robust data are needed to confirm that this technique may become a potential alternative to invasive coronary angiography for ISR detection in daily clinical practice. The most important features of a clinical test are few false negative and false positive results (affecting sensitivity and specificity, respectively). Additionally, when restenosis rate is low, as it is currently the case for simple lesions treated with DES, a high negative predictive value (NPV) allows exclude ISR in the majority of patients. Subsequently, only in a limited number of patients, invasive coronary angiography would be required to confirm and potentially treat ISR.

In order to determine the current diagnostic accuracy of 64-MDCT, we performed a meta-analysis of all available studies comparing 64-MDCT with invasive coronary angiography for the diagnosis of ISR.

\section{METHODS}

\section{Search Strategy}

Database searches for English articles published until April 2009 were performed in MEDLINE, Cochrane library, and BioMed Central databases. We combined the medical subject headings for computed tomography, multi-detector computed tomography, and coronary angiography, with the exploded terms stent and restenosis and scanned references in retrieved articles and reviews. The retrieved studies were carefully examined to exclude potential duplicates or overlapping data. Meeting abstracts were excluded, as they could not provide adequately detailed data and their results might not be final. Only papers evaluating the presence of ISR by both invasive coronary angiography and 64-MDCT in the same subjects were included.

\section{Study Eligibility}

We included a study if: (1) 64-MDCT was used as a diagnostic test for ISR, with $>50 \%$ diameter stenosis selected as the cut-off criterion for significant restenosis, using invasive coronary angiography and quantitative coronary angiography as the standard of reference; (2) absolute numbers of true positive (TP), false positive (FP), true negative (TN), and false negative $(\mathrm{FN})$ results were provided or could be derived. Studies were excluded if they were performed: (1) only in patients after coronary artery bypass graft surgery; (2) in a subset of patients with prior heart transplantation.

\section{Data Extraction}

The following information was extracted from each study: first author, year of publication, and journal; study population characteristics, including sample size (number of subjects evaluated with both tests, number of patients excluded); number of stents evaluated (and excluded from the analysis); gender; mean age (and standard deviation); mean heart rate (and standard deviation); relative timing of the two imaging procedures and whether or not evaluation of one test was blind to the result of the other; technical characteristics of the MDCT, including type and manufacturer of MDCT equipment used, and rate of beta-blocker usage. Two investigators performed data extraction independently. Discrepancies were solved by a third investigator and global consensus. The study quality conformed to the QUADAS guidelines. ${ }^{8}$

\section{Statistical Analysis}

The main analyses were performed using the traditional meta-analytic methods for combining data for diagnostic accuracy tests. ${ }^{9,10}$ The analyses that were performed to compare accuracy of 64-MDCT vs invasive coronary angiography as reference incorporated all accuracy tests separately: sensitivity, specificity, likelihood ratios, diagnostic odds ratio, and finally summarizing data in receiver operating characteristic curves (sROC). Accordingly, the absolute numbers of TP, FP, $\mathrm{TN}$, and FN findings were analyzed to provide sensitivity and specificity. Positive and negative likelihood ratios (LR+ and $\mathrm{LR}-)$ were calculated as $\mathrm{LR}+=\operatorname{sensitivity} /(1-$ specificity $)$ and $\mathrm{LR}-=(1-$ sensitivity $) /$ specificity, respectively. The $\mathrm{LR}+$ is a measure of how much the odds of the disease increase when a test is positive, while the LR - is a measure of how much the odds of the disease decrease when a test is negative. Thus, the combined likelihood ratios provides the diagnostic odds ratio (=[sensitivity/( 1 - specificity)]/ $[(1-$ specificity/sensitivity $]=($ true positive $\times$ true negative $) /$ (false positive $\times$ false negative)). The result is a ratio of the odds of a positive test result among diseased to the odds of a positive test result among nondiseased. ${ }^{9,10}$ Diagnostic odds 
ratio converts each pair of sensitivity and specificity into a single global accuracy measure.

Sensitivity and specificity were pooled as weighted means in which the weight of each study is its sample size. Likelihood ratios and diagnostic odds ratios were pooled by the DerSimonian and Laird method (random-effects model). ${ }^{11}$ The accuracy tests were repeated with inclusion of unassessable stents. The summary ROC analysis, that represents a more general method for the meta-analysis of diagnostic studies than those described above, was computed using the Moses-Shapiro-Littenberg method. ${ }^{12}$

Source of heterogeneity were explored performing a meta-regression using the Moses-Shapiro-Littenberg method with covariates ${ }^{12}$ : average age of patients, percentage of male, percentage of not evaluable stents, sample size.

Meta-analysis software package Meta-disc version 1.4 (Unit of clinical biostatics, the Ramo y Cajal Hospital, Madrid, Spain) was used for analyses. Moreover, prevalence of ISR, positive, and negative predictive values were calculated as well.

Finally, to check how robust our findings were to model specification we also performed a sensitivity analysis. Rather than using a single accuracy endpoint per study we used a multivariate meta-analysis model. We used the hierarchical summary ROC model which in our setting represents a different parametrization of the bivariate random-effects metaanalysis model. ${ }^{13}$ Additional analysis was conducted using STATA/SE 10.1 (Stata Statistical Software: Release 10. College Station, TX: StataCorp LP).

\section{RESULTS}

\section{Search Results}

The search resulted in detection of 211 hits. After the exclusion of nonrelevant articles by title and abstract, 37 articles were retrieved for full text evaluation, and of these 10 studies fulfilled all inclusion criteria. One study was excluded because it was performed using a dual-source 64-slice MDCT system. ${ }^{14}$

From the study by Rist et $\mathrm{al}^{15}$ the data on proximal, distal, and in-stent restenosis were combined to a single result. Despite using a 40-MDCT system, the study by Gaspar et $\mathrm{al}^{16}$ was included as the spatial resolution was similar to 64-MDCT. Finally, for this reason, the study by Van Mieghem et al, ${ }^{17}$ which used both 16 - and 64-slice scanners, was also included in the analysis. Accordingly, nine eligible studies ${ }^{15-23}$ were included in the meta-analysis (Table 1).

\section{Characteristics of the Included Studies}

The mean patient age was 61 years with a range of 58-68 years. Males constituted $81 \%$ (range 63-92\%), and $17 \%$ (range 12-27\%) had diabetes mellitus. The mean heart rate during data acquisition (reported in six

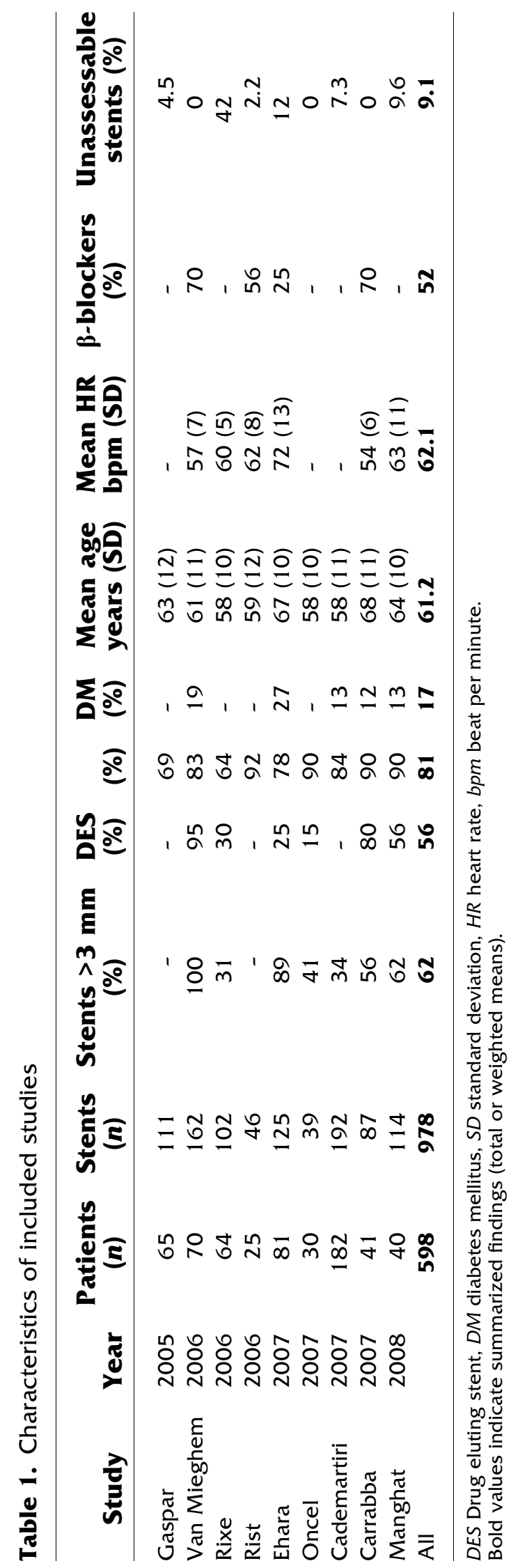


studies) was 62 beats per min with a range of 54-72 beats per min. In five studies patients received additional beta-blockers to reduce heart rate before the examination (Table 1).

\section{Stent Assessability}

The analysis included 598 patients with a total of 978 stents. The percent of DES evaluated was $56 \%$ (data available in six studies). Overall the percent of unassessable stents was 9\% (range 0-42\%), although in three studies all stented segments were evaluated ${ }^{17,19,22}$ (Table 1).

\section{Results of the Accuracy Analyses}

After the exclusion of unassessable stents, accuracy tests with 95\% confidence intervals (CIs) comparing 64-MDCT vs invasive coronary angiography for binary ISR detection showed that pooled sensitivity, specificity, positive likelihood ratio, negative likelihood ratio, and a diagnostic odds ratio (random effect model) values were $86 \%$ (95\% CI $80-91 \%$ ) (Figure 1A), 93\% (95\% CI 91-95\%) (Figure 1B), 12.32 (95\% CI 7.26-20.92) (Figure 2A), 0.18 (95\% CI 0.12-0.28) (Figure 2B), and 88.9 (95\% CI 34.6-228.6), respectively. The area under the sROC curve was 0.94 for per-stent analysis, indicating a high degree of agreement between 64-MDCT and invasive coronary angiography for ISR detection (Figure 3). Visual inspection of Figures 1 and 2 identified the presence of heterogeneity between studies. However, heterogeneity was found statistically significant for specificity $\left(\chi^{2}=25.81, \mathrm{df}=8, P=.0011\right)$, and positive likelihood ratio $\left(\chi^{2}=24.91, \mathrm{df}=8, P=\right.$ .002 ). The calculated positive and negative predictive values were $70.4 \%$ and $97.2 \%$, respectively. When including unassessable stents, accuracy tests showed that value of the pooled sensitivity did not change $(86 \%$, 95\% CI $80-91 \%$ ), whereas the pooled specificity only slightly decreased $(91 \%, 95 \%$ CI $87-92 \%)$.

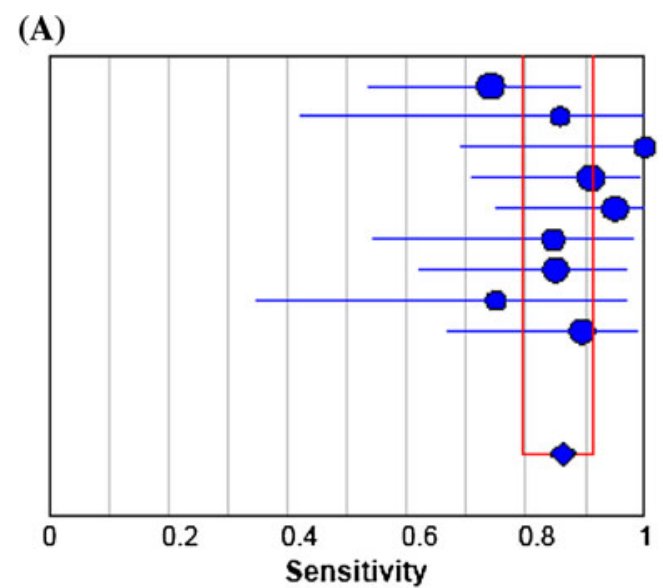

\begin{tabular}{lrc} 
& \multicolumn{3}{c}{ Sensitivity $(95 \% \mathrm{CI})$} \\
Gaspar 2005 & 0.74 & $(0.54-0.89)$ \\
Rixe 2006 & 0.86 & $(0.42-1.00)$ \\
Van Mieghem 2006 & 1.00 & $(0.69-1.00)$ \\
Ehara 2007 & 0.91 & $(0.71-0.99)$ \\
Cademartiri 2007 & 0.95 & $(0.75-1.00)$ \\
Carrabba 2007 & 0.85 & $(0.55-0.98)$ \\
Manghat 2008 & 0.85 & $(0.62-0.97)$ \\
Rist 2006 & 0.75 & $(0.35-0.97)$ \\
Oncel 2007 & 0.89 & $(0.67-0.99)$
\end{tabular}

(B)

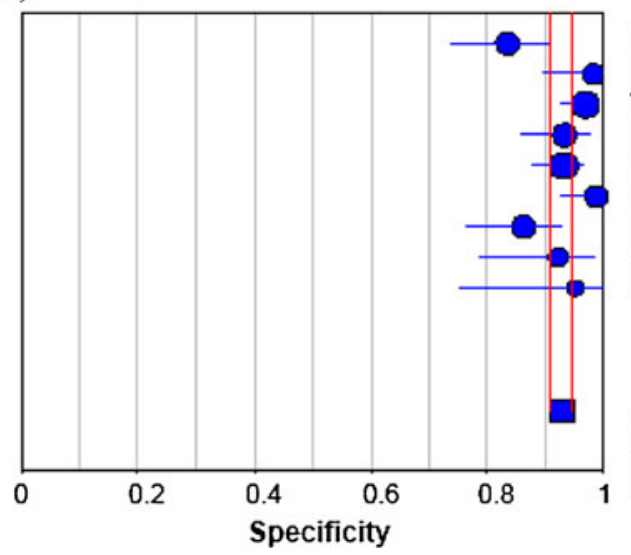

Gaspar 2005

Rixe 2006

Van Mieghem 2006

Ehara 2007

Cademartiri 2007

Carrabba 2007

Manghat 2008

Rist 2006

Oncel 2007

Specificity $(95 \% \mathrm{Cl})$

$0.83(0.74-0.91)$

$0.98 \quad(0.90-1.00)$

$0.97 \quad(0.92-0.99)$

$0.93(0.86-0.97)$

$0.93(0.88-0.96)$

$0.99 \quad(0.93-1.00)$

$0.86 \quad(0.76-0.93)$

$0.92(0.79-0.98)$

$0.95(0.75-1.00)$

Figure 1. (A) Plot and table of in-stent restenosis detection sensitivity of 64-MDCT vs invasive coronary angiography; (B) plot and table of ISR detection specificity of 64-slice multi-detector computed tomography vs invasive coronary angiography. 


\section{Heterogeneity Analyses}

The meta-regression analysis to explore the reasons of heterogeneity, including all important covariates, which we presumed to be the most likely source of heterogeneity, did not show any significant effect. Quality assessment for all included studies is shown in Table 2 .

\section{Additional Analyses}

Results from the bivariate meta-analysis model show negligible differences. Sensitivity, specificity, positive and negative likelihood ratios, and diagnostic odds ratio pooled estimates were 88 (95\% CI 80-93\%), 94\% (95\% CI 90-96\%), 13.8 (95\% CI 8.2-23.2), 0.13 (95\% C 0.1-0.2), and 107.20 (95\% CI 42.9-267.9), respectively.

\section{DISCUSSION}

In this meta-analysis, we focused on the diagnostic performance of the 64-MDCT generation of MDCT as a potential alternative, noninvasive method for ISR detection. We observed an average weighted sensitivity of $86 \%$ and specificity of $93 \%$ for $64-\mathrm{MDCT}$ in the detection of ISR as compared to invasive coronary angiography. These observations are not substantially different from the meta-analysis by Hamon et al, who included both 16- and 64-MDCT systems. ${ }^{24}$ While in fact the majority of data were derived from the older 16-MDCT systems, only slightly lower average weighted sensitivity (84\%) and specificity (91\%) were reported. However, one should realize that both in the meta-analysis of Hamon et $\mathrm{al}^{24}$ as well as the current one, these values were obtained after exclusion of uninterpretable stents. Possibly, the main advantage of improved temporal and spatial resolution may lie in improved rates of interpretable stents. Indeed, with previous generations of MDCT scanners, diagnosis of ISR was particularly limited by the high percentages of stents with insufficient image quality for evaluation. Based on in vitro studies as well as initial clinical investigations, improvement in the rate of interpretable stents has been anticipated with the introduction of 64MDCT with higher spatial and temporal resolution. ${ }^{15,25}$
(A)

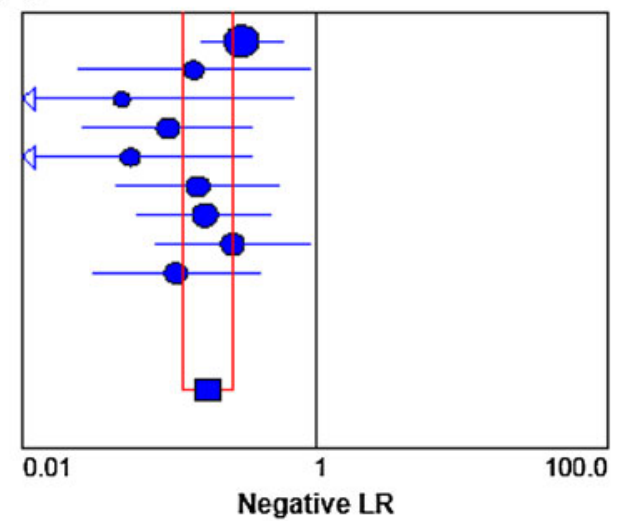

(B)

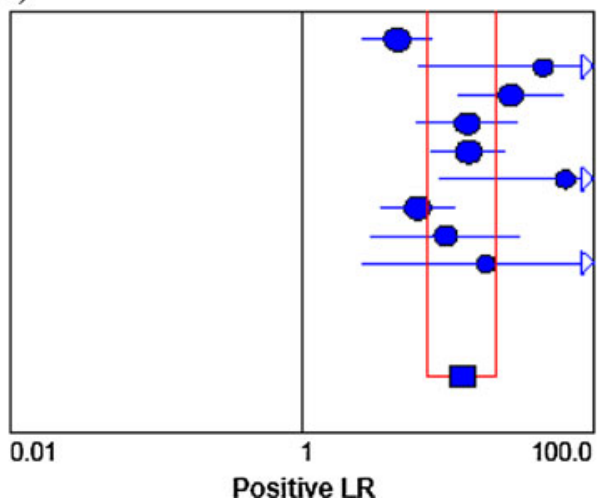

Negative LR $(95 \% \mathrm{CI})$

Gaspar 2005

Rixe 2006

Van Mieghem 2006

Ehara 2007

Cademartiri 2007

Carrabba 2007

Manghat 2008

Rist 2006

Oncel 2007

\section{Random Effects Model}

Pooled Negative LR $=0.18(0.12$ to 0.28$)$

Cochran- $Q=8.34 ; \mathrm{df}=8(\mathrm{p}=0.4010)$

Inconsistency (I-square) $=4.1 \%$

Tau-squared $=0.0170$

\section{Gaspar 2005}

Rixe 2006

Van Mieghem 2006

Ehara 2007

Cademartiri 2007

Carrabba 2007

Manghat 2008

Rist 2006

Oncel 2007

Positive LR $(95 \% \mathrm{Cl})$

$4.44 \quad(2.62-7.53)$

$44.57 \quad(6.25-317.84)$

$26.55(11.57-60.94)$

$13.33(6.09-29.19)$

$13.65(7.65-24.34)$

$62.62(8.82-444.72)$

$6.10(3.42-10.89)$

$9.50 \quad(2.99-30.22)$

$17.89(2.63-121.64)$

Random Effects Model

Pooled Positive LR = 12.32 (7.26 to 20.92)

Cochran-Q = 24.91; df $=8(p=0.0016)$

Inconsistency (l-square) $=67.9 \%$

Tau-squared $=0.3787$

Figure 2. (A) Plot and table of in-stent restenosis detection negative likelihood ratio of 64-MDCT vs invasive coronary angiography; (B) plot and table of in-stent restenosis detection positive likelihood ratio of 64-slice multi-detector computed tomography vs invasive coronary angiography. 


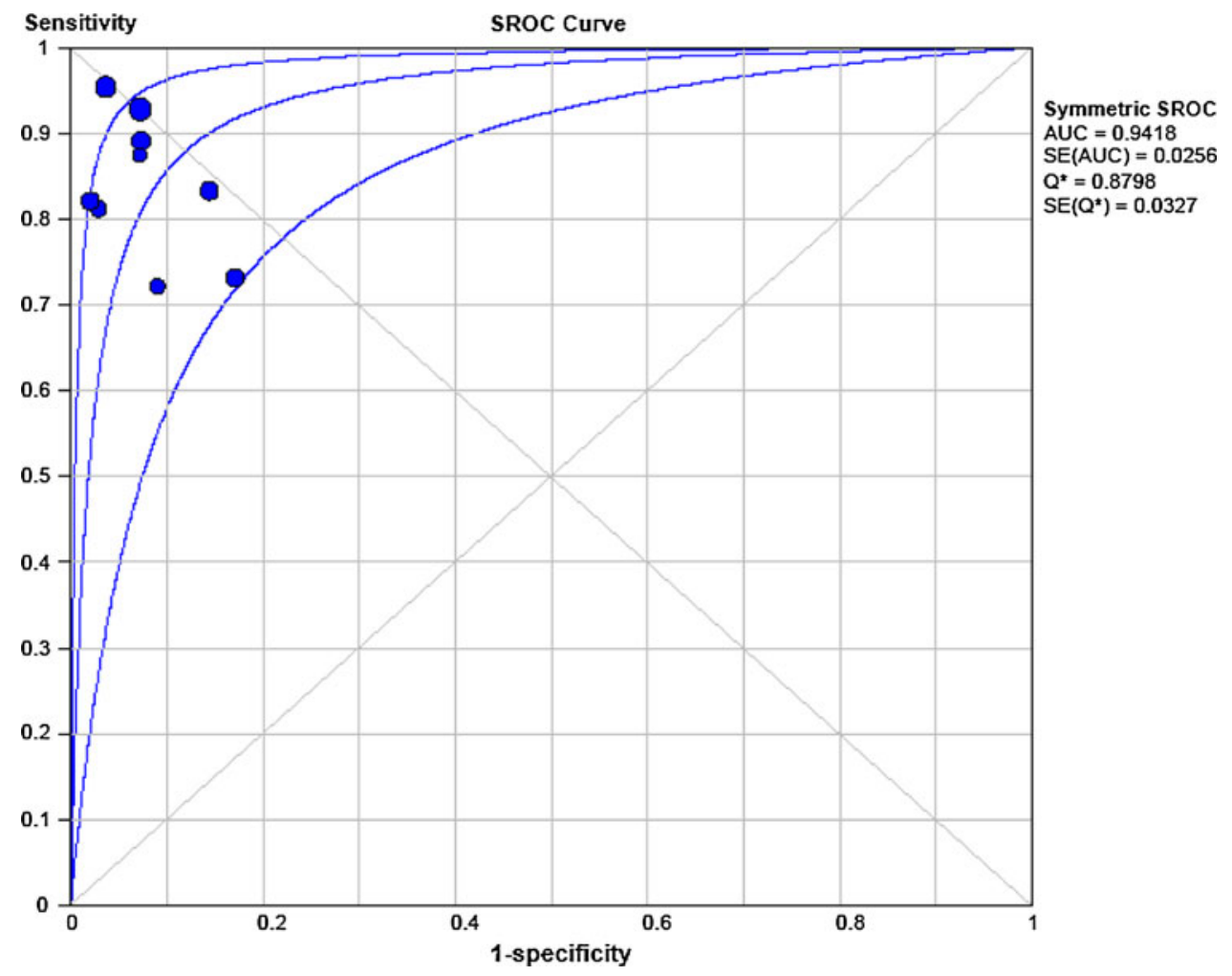

Figure 3. Receiver operator characteristic (ROC) curve analysis on a per stented segment basis (nine studies) comparing 64-MDCT vs invasive coronary angiography. The diagnostic accuracy is shown by plotting 1-specificity against sensitivity, area under curve (AUC), and $Q^{*}$ statistic with their standard errors (SE). The upper and lower lines indicate 95\% CIs. SROC Summary receiver operating characteristics.

However, thus far no robust data were available to support this expectation. Previous meta-analyses addressing the value of MDCT in the detection of ISR still reported high rates of uninterpretable stents of approximately $13 \% .{ }^{24,26}$ Importantly, these meta-analyses, as described above, included both 16- and 64-MDCT with the majority of data derived from the older 16-MDCT systems. In contrast, the current analysis was restricted to 64-MDCT and showed a relatively slight reduction in the rate of nonassessable stents (9\%) as compared to these previous meta-analyses (absolute reduction of 4.4\%; relative reduction of $30 \%$ ). Accordingly, the current analysis supports that the use of 64-MDCT may indeed result in improved stent assessability.

However, despite this improvement as compared to previous investigations, the rate of uninterpretable stents was still not negligible. Moreover, the majority of included studies used strict inclusion criteria with exclusion of patients with smaller stents, contraindication to administration of beta-blocking medication, elevated heart rate and obesity. In previous studies, these factors have been shown to negatively influence stent evaluation with MDCT. ${ }^{7,27,28}$ Indeed, as can be derived from Table 1, also in the currently included studies, rates of uninterpretable stents tended to be higher in the presence of a higher mean heart rate, no additional administration of beta-blocking medication or a relatively higher proportion of stents with a diameter $<3.0 \mathrm{~mm}$. Of note, the results of the present metaanalyses showed significant heterogeneity between the included studies. Thus, our results should be interpreted cautiously, although the use of the random-effects model and the bivariate random-effects meta-analysis model should at least partly correct for this issue. Exploring sources of heterogeneity by meta-regression analyses showed that age, percentage of male, percentage of nonevaluable stents and sample size had no significant influence. Possible sources of heterogeneity were a significant difference in the numbers of assessed stents between studies and the use of kernel convolution filters, which might have influenced the absolute numbers of true and false ISR reported in each study. These variations in-stent analysis may be attributed to the level of experience with 64-MDCT in different study centers. Improvement of technical quality and clinical experience might minimize this difference. 


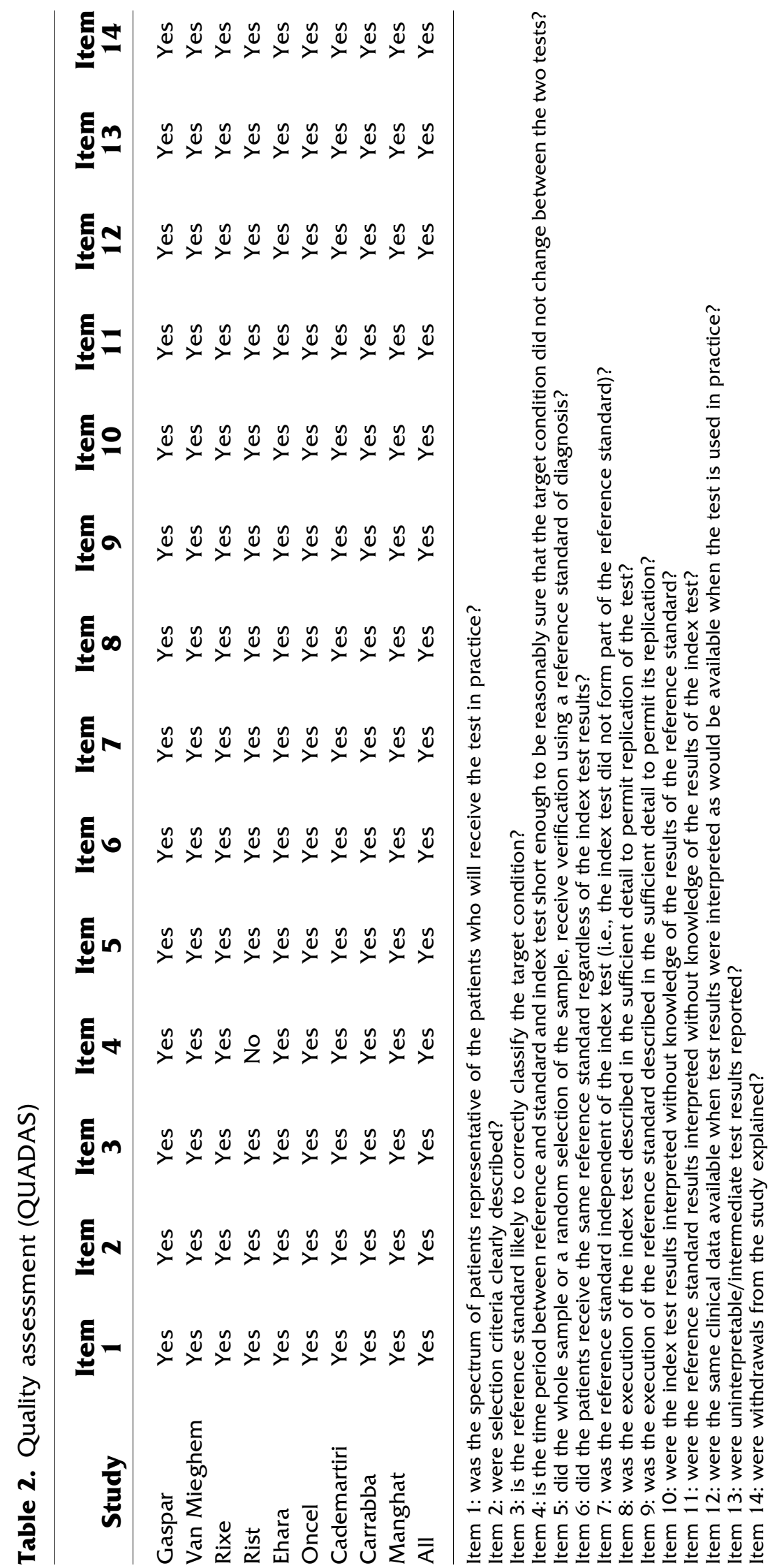




\section{Clinical Implications}

The current meta-analysis confirms that in selected patients, 64-MDCT can reliably rule out ISR. The high NPV of $97.2 \%$ indicates that if stent patency is demonstrated on 64-MDCT, further evaluation by means of invasive coronary angiography can be avoided. Accordingly, the technique may be a particularly suitable alternative for patients without a high clinical suspicion of ISR and with expected low rate of ISR, but in whom further clinical evaluation is still preferred. However, patients with a high likelihood of ISR, including patients with typical chest pain and/or an unambiguously abnormal stress test, are unlikely to benefit from initial noninvasive evaluation with MDCT. In this patient population, MDCT is limited by its low positive predictive value, inability to determine the hemodynamical relevance of detected lesions and the fact that no immediate intervention can be performed. Accordingly, use of MDCT may only result in unnecessary radiation exposure, although recent developments in technology and software currently allow coronary CT angiography with estimated doses $<5 \mathrm{mSv}^{29,30}$ In addition, MDCT imaging remains unsuitable for patients with characteristics unfavorable for stent imaging, such as small stents or elevated heart rate. Indeed, it remains important to realize that even with 64-MDCT only in selected patients, the high diagnostic accuracy and relatively low rates of uninterpretable stents are maintained. Routine use of 64-MDCT to rule out ISR in the general population therefore, cannot be recommended. Potentially, improved rates of stent interpretation may be achieved in the near future with the use of dual-source systems with higher temporal resolution, ${ }^{14}$ high-definition or flat panel detector scanners with improved spatial resolution ${ }^{31,32}$ or even 320-row systems that allow cardiac imaging in a single volume. ${ }^{33}$

\section{CONCLUSIONS}

The results of the current meta-analysis demonstrate that 64-MDCT has a good diagnostic accuracy for ISR detection with a particularly high NPV. However, still a relatively large proportion of stents, although improved as compared to previous reports, remains uninterpretable. Accordingly, only in selected patients, 64-MDCT may serve as a potential alternative noninvasive method to rule out ISR.

\section{Acknowledgments}

We are grateful to Annibale Biggeri, from the Biostatistics Unit, Cancer Prevention and Research Institute ISPO, Florence, for his assistance with the statistical analyses performed for this research.

\section{Open Access}

This article is distributed under the terms of the Creative Commons Attribution Noncommercial License which permits any noncommercial use, distribution, and reproduction in any medium, provided the original author(s) and source are credited.

\section{References}

1. Fischman DL, Leon MB, Baim DS, et al. A randomized comparison of coronary-stent placement and balloon angioplasty in the treatment of coronary artery disease. Stent Restenosis Study Investigators. N Engl J Med 1994;331:496-501.

2. Serruys PW, de Jaegere P, Kiemeneij F, et al. A comparison of balloon-expandable-stent implantation with balloon angioplasty in patients with coronary artery disease. Benestent Study Group. N Engl J Med 1994;331:489-95.

3. Stone GW, Ellis SG, Cox DA, et al. A polymer-based, paclitaxeleluting stent in patients with coronary artery disease. $\mathrm{N}$ Engl $\mathrm{J}$ Med 2004;350:221-31.

4. Morice MC, Serruys PW, Sousa JE, et al. A randomized comparison of a sirolimus-eluting stent with a standard stent for coronary revascularization. N Engl J Med 2002;346:1773-80.

5. Zellweger MJ, Weinbacher M, Zutter AW, et al. Long-term outcome of patients with silent versus symptomatic ischemia six months after percutaneous coronary intervention and stenting. $\mathbf{J}$ Am Coll Cardiol 2003;42:33-40.

6. Nieman K, Cademartiri F, Lemos PA, et al. Reliable noninvasive coronary angiography with fast submillimeter multislice spiral computed tomography. Circulation 2002;106:2051-4.

7. Schuijf JD, Bax JJ, Jukema JW, et al. Feasibility of assessment of coronary stent patency using 16-slice computed tomography. Am J Cardiol 2004;94:427-30.

8. Whiting P, Harbord R, Kleijnen J. No role for quality scores in systematic reviews of diagnostic accuracy studies. BMC Med Res Methodol 2005;5:19.

9. Deeks JJ. Systematic reviews in health care: Systematic reviews of evaluations of diagnostic and screening tests. BMJ 2001;323:15762.

10. Deville WL, Buntinx F, Bouter LM, et al. Conducting systematic reviews of diagnostic studies: Didactic guidelines. BMC Med Res Methodol 2002;2:9.

11. DerSimonian R, Laird N. Meta-analysis in clinical trials. Control Clin Trials 1986;7:177-88.

12. Moses LE, Shapiro D, Littenberg B. Combining independent studies of a diagnostic test into a summary ROC curve: Dataanalytic approaches and some additional considerations. Stat Med 1993;12:1293-316.

13. Harbord RM, Deeks JJ, Egger M, et al. A unification of models for meta-analysis of diagnostic accuracy studies. Biostatistics 2007;8:239-51.

14. Pugliese F, Weustink AC, van Mieghem C, et al. Dual source coronary computed tomography angiography for detecting in-stent restenosis. Heart 2008;94:848-54.

15. Rist C, von Ziegler F, Nikolaou K, et al. Assessment of coronary artery stent patency and restenosis using 64-slice computed tomography. Acad Radiol 2006;13:1465-73.

16. Gaspar T, Halon DA, Lewis BS, et al. Diagnosis of coronary instent restenosis with multidetector row spiral computed tomography. J Am Coll Cardiol 2005;46:1573-9. 
17. van Mieghem CA, Cademartiri F, Mollet NR, et al. Multislice spiral computed tomography for the evaluation of stent patency after left main coronary artery stenting: A comparison with conventional coronary angiography and intravascular ultrasound. Circulation 2006;114:645-53.

18. Cademartiri F, Schuijf JD, Pugliese F, et al. Usefulness of 64-slice multislice computed tomography coronary angiography to assess in-stent restenosis. J Am Coll Cardiol 2007;49:2204-10.

19. Carrabba N, Bamoshmoosh M, Carusi LM, et al. Usefulness of 64slice multidetector computed tomography for detecting drug eluting in-stent restenosis. Am J Cardiol 2007;100:1754-8.

20. Ehara M, Kawai M, Surmely JF, et al. Diagnostic accuracy of coronary in-stent restenosis using 64-slice computed tomography: Comparison with invasive coronary angiography. J Am Coll Cardiol 2007;49:951-9.

21. Manghat N, Van Lingen R, Hewson P, et al. Usefulness of 64detector row computed tomography for evaluation of intracoronary stents in symptomatic patients with suspected in-stent restenosis. Am J Cardiol 2008;101:1567-73.

22. Oncel D, Oncel G, Karaca M. Coronary stent patency and in-stent restenosis: Determination with 64-section multidetector CT coronary angiography_initial experience. Radiology 2007;242:403-9.

23. Rixe J, Achenbach S, Ropers D, et al. Assessment of coronary artery stent restenosis by 64-slice multi-detector computed tomography. Eur Heart J 2006;27:2567-72.

24. Hamon M, Champ-Rigot L, Morello R, et al. Diagnostic accuracy of in-stent coronary restenosis detection with multislice spiral computed tomography: A meta-analysis. Eur Radiol 2008;18:217-25.

25. Mahnken AH, Muhlenbruch G, Seyfarth T, et al. 64-slice computed tomography assessment of coronary artery stents: A phantom study. Acta Radiol 2006;47:36-42.
26. Sun Z, Almutairi AM. Diagnostic accuracy of 64 multislice CT angiography in the assessment of coronary in-stent restenosis: A meta-analysis. Eur J Radiol 2010;73:266-73.

27. Mahnken AH, Buecker A, Wildberger JE, et al. Coronary artery stents in multislice computed tomography: In vitro artifact evaluation. Invest Radiol 2004;39:27-33.

28. Nieman K, Rensing BJ, van Geuns RJ, et al. Non-invasive coronary angiography with multislice spiral computed tomography: Impact of heart rate. Heart 2002;88:470-4.

29. Hausleiter J, Meyer T, Hadamitzky M, et al. Radiation dose estimates from cardiac multislice computed tomography in daily practice: Impact of different scanning protocols on effective dose estimates. Circulation 2006;113:1305-10.

30. Stolzmann P, Leschka S, Scheffel H, et al. Dual-source CT in stepand-shoot mode: Noninvasive coronary angiography with low radiation dose. Radiology 2008;249:71-80.

31. Mahnken AH, Seyfarth T, Flohr T, et al. Flat-panel detector computed tomography for the assessment of coronary artery stents: Phantom study in comparison with 16-slice spiral computed tomography. Invest Radiol 2005;40:8-13.

32. Min JK, Swaminathan RV, Vass M, et al. High-definition multidetector computed tomography for evaluation of coronary artery stents: Comparison to standard-definition 64-detector row computed tomography. J Cardiovasc Comput Tomogr 2009;3:246-51.

33. de Graaf FR, Schuijf JD, van Velzen JE et al. Diagnostic Accuracy of 320-Row Multidetector Computed Tomography Coronary Angiography to Non-Invasively Assess In-Stent Restenosis. Invest Radiol 2010, in press. 